\title{
Alcoolismo e tabagismo em idosos: relação com ingestão alimentar e aspectos socioeconômicos
}

Alcoholism and smoking in the elderly: relation to dietary intake and socioeconomic aspects

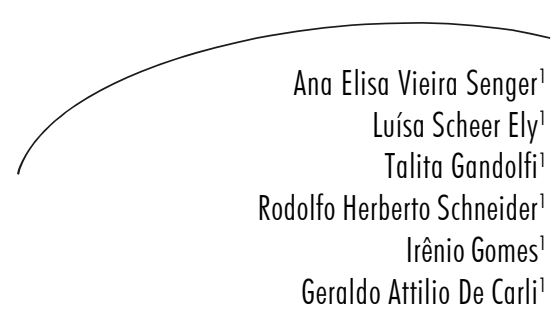

Resumo

Objetivos: Verificar a prevalência do alcoolismo e tabagismo em idosos residentes na cidade de Porto Alegre, RS, e estabelecer sua relação entre a ingestão alimentar diária e o nível socioeconômico, de acordo com o sexo, grupo etário, escolaridade, renda, situação e composição familiar. Métodos: Esta pesquisa fez parte do Projeto Estudo Multidimensional dos Idosos de Porto Alegre (EMIPOA), que foi desenvolvida pelo IGG/PUCRS. As entrevistas foram realizadas no ambulatório do Instituto de Geriatria e Gerontologia do Hospital São Lucas da PUCRS. Foi aplicado um questionário com dados socioeconômicos e de saúde por profissionais treinados. Resultados: Dos 832 entrevistados, $71,2 \%$ eram mulheres e $28,8 \%$ homens. A faixa etária mais prevalente foi de 60 a 69 anos (44,8\%). Com prevalência na faixa etária acima citada de 7,5\% para alcoolistas e $23,9 \%$ para tabagistas. O alcoolismo e o tabagismo prevaleceram entre os homens, $11,7 \%$ e $20,8 \%$, respectivamente. Em relação à escolaridade, a maior prevalência foi entre alcoolistas e tabagistas que possuíam apenas o $1^{\circ}$ grau, $4,7 \%$ e $16,1 \%$, respectivamente. Observou-se que dentre os idosos alcoolistas ou fumantes, a maioria não tem relação familiar $(P<0,001)$. Já em relação à ingestão alimentar, houve um maior número de fumantes ou alcoolistas que fazem de 1 a 2 refeições diárias. Conclusões: Existem poucos estudos que investigam as tendências e consequências do tabagismo e alcoolismo entre a população idosa. Ações de saúde devem ser planejadas, visando a diminuir o consumo abusivo de álcool e tabagismo, bem como prevenir seus malefícios a saúde.

\footnotetext{
Instituto de Geriatria e Gerontologia. Curso de Pós-Graduação em Gerontologia Biomédica. Pontifícia Universidade Católica do Rio Grande do Sul-PUCRS.

Resumo apresentado no Simpósio Sul-Americano de Geriatria e Gerontologia, Porto Alegre-RS, 30 julho a 01 agosto de 2009 .

Correspondência / Correspondence

Talita Gandolfi

Rua Felix da Cunha, 667 - Floresta

90570-001 Porto Alegre, RS, Brasil

E-mail: talita.gandolfi@acad.pucrs.br
}

Palavras-chave: Idosos. Alcoolismo. Tabagismo. Alimentação. Ingestão Alimentar. 


\section{Abstract}

Objectives: To determine the prevalence of alcoholism and smoking among the elderly living in Porto Alegre city and to establish an association between daily food intake and socioeconomic status according to sex, age group, income, location and family composition. Methods: This research was conducted as Multidimensional Study of the Elderly in Porto Alegre city (EMIPOA), which was developed by the IGG/PUCRS. The interviews were conducted in the ambulatory of the Institute of Geriatrics and Gerontology, Hospital São Lucas of PUCRS. We administered a questionnaire with socioeconomic data and health data by trained professionals. Results: Of the 832 respondents, $71.2 \%$ were women and $28.8 \%$ men. The most prevalent age range was 60 to 69 years $(44.8 \%)$. Prevalence in the age group above-mentioned $7.5 \%$ for alcoholics and $23.9 \%$ for smokers. Alcoholism and smoking prevailed among men, $11.7 \%$ and $20.8 \%$ respectively. Regarding education, the highest prevalence was among alcoholics and smokers who had only 1 degree, $4.7 \%$ and $16.1 \%$, respectively. It was observed that among the elderly alcoholics or smokers, most do not have family relationship $(P<0.001)$. In relation to food ingestion, there was higher number of smokers or alcoholics that are 1 to 2 daily meals. Conclusions: There are few studies investigating the trends and consequences of smoking and alcoholism among the elderly. Health actions should be planned in order to decrease the abuse of alcohol and smoking, and prevent its harm to health.
Key words: Elderly. Alcoholism. Smoking. Food. Eatin.

\section{INTRODUÇÃO}

O envelhecimento populacional está ocorrendo de forma rápida, principalmente em países em desenvolvimento, levando a importantes desafios sociais e econômicos. A prevalência de doenças crônico-degenerativas reforça a necessidade de ações eficazes no sentido de promover melhor qualidade de vida aos idosos. ${ }^{1}$

Embora o envelhecimento seja um processo natural, leva o organismo a várias alterações anatômicas e funcionais, com repercussões nas condições de saúde e nutrição do idoso. ${ }^{2}$ Associadas às alterações decorrentes do envelhecimento, mudanças como aposentadoria, perda de amigos, solidão e isolamento social, deixam os idosos vulneráveis e mais propensos a intensificação de hábitos menos saudáveis, como o consumo abusivo de álcool e o tabagismo. ${ }^{3}$

\section{Alcoolismo e tabagismo no idoso}

De acordo com alguns estudos epidemiológicos, cerca de um terço da população idosa começa a fazer uso do álcool tardiamente.
São comuns os problemas relacionados ao abuso do álcool em indivíduos maiores de 60 anos, mas por serem pouco reconhecidos são denominados por alguns autores de "epidemia invisível". ${ }^{4}$

Estudos realizados em amostras clínicas evidenciaram um aumento significativo do uso de álcool na população idosa. As pesquisas mostram que 6 a $11 \%$ dos pacientes idosos admitidos em hospitais gerais apresentam sintomas de dependência alcoólica, inclusive as estimativas de admissão por alcoolismo nos serviços de emergência se equiparam às admissões por infarto. Apesar disto, a equipe hospitalar reconhece menos casos de alcoolismo em idosos do que em pacientes mais jovens. ${ }^{5}$

O consumo excessivo de álcool interfere de várias maneiras na nutrição adequada do idoso, pois compete com os nutrientes desde sua ingestão até sua absorção e utilização. Quanto maior a participação do álcool na dieta, menor a densidade e qualidade nutricional da alimentação. ${ }^{4}$

O tabagismo é considerado um relevante problema de saúde pública em todo o mundo. As estimativas são de que $1 / 3$ da população 
mundial adulta seja fumante, com prevalência de $47 \%$ na população masculina e $12 \%$ na população feminina. A mortalidade mundial, por doenças associadas ao fumo, atinge cerca de 4,9 milhões de mortes por ano, o que representa 10 mil mortes por dia. ${ }^{6} \mathrm{O}$ hábito de fumar está particularmente associado em usuários de bebidas alcoólicas, predispondo o indivíduo a importantes alterações na capacidade visual e cognitiva, causando sofrimento pessoal, familiar e alto custo social. ${ }^{6,7}$

Além da nicotina, um alcalóide com propriedades psicoativas, existem no cigarro mais de 4.500 substâncias químicas com efeitos cancerígenos, mutagênicos, tóxicos e irritantes. ${ }^{8}$ O consumo de tabaco está associado ao aumento do risco de diversas doenças do aparelho gastrointestinal, designadamente: câncer do esôfago, do estômago, do pâncreas, do fígado e do cólon, refluxo gastroesofágico, úlcera péptica gástrica e duodenal e doença de Chron. ${ }^{9}$

O tabagismo influencia também nos processos metabólicos, pois fumantes geralmente pesam em média $4 \mathrm{~kg}$ a menos que não fumantes; alterações essas resultantes de um aumento da taxa metabólica concomitante a uma supressão do apetite. $^{10}$

Diante do exposto, este artigo tem por objetivo verificar a prevalência do alcoolismo e tabagismo em idosos residentes em Porto Alegre e estabelecer sua relação entre a ingestão alimentar diária e o nível socioeconômico, de acordo com o sexo, grupo etário, escolaridade, renda, situação e composição familiar.

\section{METODOLOGIA}

Este é um estudo transversal de base populacional em idosos (60 anos ou mais) da cidade de Porto Alegre, Rio Grande do Sul, Brasil. Esta pesquisa fez parte do Projeto Estudo Multidimensional dos Idosos de Porto Alegre (EMIPOA), que foi desenvolvida pelo IGG/ PUCRS. O tamanho da amostra foi calculado em 1.092 idosos, baseando-se no número de indivíduos avaliados em estudo anterior (1995), para cada bairro da cidade de Porto Alegre, atualizado pelo Instituto Brasileiro de Geografia e Estatística (IBGE), de acordo com as estimativas de variação populacional para 2005. Os entrevistados foram recrutados de forma estratificada por região censitária, sendo sorteada a primeira residência e escolhidas as demais de forma sistemática. Foi aplicado um questionário com dados socioeconômicos e de saúde por profissionais treinados do Serviço Social da PUCRS, nas residências escolhidas.

Neste estudo, relacionaram-se as respostas das perguntas direcionadas para o uso de álcool e cigarro, situação familiar, dados socioeconômicos e número de refeições diárias dos idosos. As perguntas relacionadas ao alcoolismo foram as do questionário SRQ-A (Self-Reporting Questionnaire). O SRQ-A é composto por cinco questões para rastreamento de transtornos pelo uso de álcool e é um questionário aprovado pelo WHO (World Health Organization).

O projeto foi aprovado pelo Comitê de Ética em Pesquisa da Pontifícia Universidade Católica do Rio Grande do Sul, conforme parecer $n^{\circ}$ 0502935, e todos os idosos assinaram o Termo de Consentimento Livre e Esclarecido antes de responderem ao questionário. Durante o estudo, foram cumpridos os princípios éticos contidos na Declaração de Helsinki. Os dados foram digitados no programa Excel e analisados com o pacote estatístico SPSS versão 17.0. A associação do alcoolismo e tabagismo com as variáveis socioeconômicas e número de refeições foi testada pelo Qui-Quadrado, considerando significativos os valores de $P$ inferiores ou iguais a 0,05.

\section{RESULTADOS}

O total de idosos que responderam a todas as questões requeridas para este estudo foi de 832 . Destes, 592 (71,2\%) eram mulheres e 240 (28,8\%) eram homens. A faixa etária mais prevalente foi a dos 60 aos 69 anos, tendo um total de $373(44,8 \%)$ idosos, seguido de 319 (38,3\%) idosos com idade entre 70 e 79 anos e 140 (16,8\%) idosos com 80 anos ou mais. A distribuição dos idosos segundo 
sexo, faixa etária, escolaridade, renda, situação e composição familiar e refeições diárias está relacionada com o uso de álcool e cigarro, conforme mostra a tabela 1.

Tabela 1 - População estudada e prevalência de alcoolismo e tabagismo, de acordo com variáveis socioeconômicas e número de refeições diárias em 832 idosos (60 anos ou mais) residentes no município de Porto Alegre, RS, 2006.

\begin{tabular}{|c|c|c|c|c|c|}
\hline VARIÁVEL & $\begin{array}{c}\text { População } \\
\text { Estudada } \\
\text { N (\%) }\end{array}$ & $\begin{array}{c}\text { Prevalência de } \\
\text { Alcoolismo } \\
(\%)\end{array}$ & $P$ & $\begin{array}{c}\text { Prevalência de } \\
\text { Tabagismo } \\
(\%)\end{array}$ & $P$ \\
\hline \multicolumn{6}{|l|}{ Sexo } \\
\hline Feminino & $592(71,2)$ & 0,7 & \multirow{2}{*}{$<0,001$} & 13,0 & \multirow{2}{*}{0,004} \\
\hline Masculino & $240(28,8)$ & 11,7 & & 20,8 & \\
\hline \multicolumn{6}{|l|}{ Idade } \\
\hline $60-69$ & $373(44,8)$ & 7,5 & \multirow{3}{*}{$<0,001$} & 23,9 & \multirow{3}{*}{$<0,001$} \\
\hline $70-79$ & $319(38,3)$ & 1,3 & & 11,9 & \\
\hline$\geq 80$ & $140(16,8)$ & 0,0 & & 0,0 & \\
\hline \multicolumn{6}{|l|}{ Renda familiar } \\
\hline Até 2 salários & $265(31,9)$ & 4,9 & \multirow{3}{*}{0,180} & 17,4 & \multirow{3}{*}{0,270} \\
\hline 2 a 5 salários & $316(38,0)$ & 4,4 & & 15,8 & \\
\hline$>5$ salários & $251(30,2)$ & 2,0 & & 12,4 & \\
\hline \multicolumn{6}{|l|}{ Escolaridade } \\
\hline Até $1^{\circ} \mathrm{grau}$ & $665(79,9)$ & 4,7 & \multirow[b]{2}{*}{0,015} & 16,1 & \multirow[b]{2}{*}{0,186} \\
\hline $2^{\circ}$ grau ou Superior & $167(20,1)$ & 0,6 & & 12,0 & \\
\hline \multicolumn{6}{|l|}{ Com quem mora } \\
\hline Com familiar & $582(70,0)$ & 4,1 & \multirow{3}{*}{0,792} & 14,8 & \multirow{3}{*}{0,195} \\
\hline Não familiar & $40(4,8)$ & 2,5 & & 7,5 & \\
\hline Sozinho & $210(25,2)$ & 3,3 & & 18,1 & \\
\hline \multicolumn{6}{|l|}{ Relação familiar } \\
\hline Satisfeito & $787(94,6)$ & 3,6 & \multirow{3}{*}{0,107} & 14,1 & \multirow{3}{*}{$<0,001$} \\
\hline Não satisfeito & $30(3,6)$ & 6,7 & & 30,1 & \\
\hline Sem relação & $15(1,8)$ & 13,3 & & 46,7 & \\
\hline \multicolumn{6}{|l|}{ Refeições/dia } \\
\hline $1-2$ & $89(10,7)$ & 5,6 & \multirow{3}{*}{0,465} & 28,1 & \multirow{3}{*}{0,004} \\
\hline $3-4$ & $622(74,8)$ & 3,4 & & 14,1 & \\
\hline $5-8$ & $121(14,5)$ & 5,0 & & 11,6 & \\
\hline TOTAL & $832(100)$ & 3,8 & --- & 15,3 & --- \\
\hline
\end{tabular}

De acordo com a análise estatística, houve uma diferença significativa quando se relacionou a variável sexo com o uso de álcool $(P<0,001)$ e cigarro $(P=0,004)$ pelos idosos. A maioria dos fumantes e alcoolistas são homens, 20,8\% e 11,7\%, respectivamente. Em relação à escolaridade, a maior prevalência foi entre alcoolistas e tabagistas que possuíam apenas o $1^{\circ}$ grau, $4,7 \%$ e $16,1 \%$, respectivamente. Outra variável significativa $(P<0,001)$ foi a faixa etária de 60-69 anos, onde se encontraram mais idosos alcoolistas $(7,5 \%)$ e fumantes $(23,9 \%)$.

A relação familiar também mostrou ser significativa $(P<0,001)$, quando relacionada com o uso abusivo de álcool e cigarro. A prevalência de fumantes e alcoolistas indicou ser maior naqueles idosos que não possuem relação familiar, $46,7 \%$ e $13,3 \%$, respectivamente. Considerando a composição familiar desses idosos, ou seja, com 
quem eles moram, não houve diferença significativa quando se verificou a prevalência de alcoolistas e fumantes, pois a mesma proporção foi encontrada naqueles idosos que moram sozinhos, com familiares ou com não familiares. Já em relação às refeições diárias, verificou-se que a prevalência de idosos alcoolistas e fumantes foi maior dentre aqueles que faziam apenas de 1 a 2 refeições diárias, 5,6\% e 28,1\%, respectivamente.

\section{DISCUSSÃO}

Poucos são os estudos que relatam o impacto do uso de álcool e cigarro na vida dos idosos, especialmente nos países em desenvolvimento, como o Brasil, onde a população idosa vem crescendo aceleradamente. ${ }^{11}$

Do ponto de vista da Saúde Pública, os cinco mais importantes fatores de risco para doenças crônicas não-transmissíveis são o tabagismo, o consumo de álcool, a obesidade ou sobrepeso, a hipertensão e a dislipidemia.

O tabaco é um dos mais potentes agentes carcinogênicos para o ser humano e seu consumo, assim como a exposição à fumaça produzida pelo fumante, é identificado como a maior causa passível de prevenção de doenças. $\mathrm{O}$ consumo aumentado de álcool está associado à hipertensão arterial, à cirrose, ao acidente vascular hemorrágico e aos cânceres da orofaringe, laringe, esôfago e fígado. ${ }^{12}$

Os dados deste estudo relatam a prevalência de idosos alcoolistas e/ ou fumantes na cidade de Porto Alegre, RS. A proporção de fumantes entre os homens $(20,8 \%)$ foi maior do que entre as mulheres (13,0\%), assim como quando relacionado com o alcoolismo, encontraram-se mais homens alcoolistas (11,7\%) do que mulheres (0,7\%). Em um estudo feito na Coreia, observou-se que a proporção de homens alcoolistas e fumantes era bem maior do que entre as mulheres. ${ }^{13}$ Outro estudo em Lubeck, Alemanha, revelou que idosos acima de 64 anos que deram entrada em hospitais foram diagnosticados como dependentes alcoólicos em $17,7 \%$ do sexo masculino e $4,2 \%$ do sexo feminino. ${ }^{14}$ Em um estudo feito em Bambuí (MG) e Belo Horizonte (MG), também encontrouse um número maior de homens idosos fumantes, $31,4 \%$ e $19,6 \%$, respectivamente, enquanto $10,3 \%$ e $8,1 \%$ das mulheres eram fumantes. $O$ autor relata que a menor prevalência do tabagismo observada entre as mulheres idosas deve ser analisada com cautela, uma vez que esse hábito tende a aumentar entre as mulheres mais jovens. ${ }^{15}$

A escolaridade também mostra estar relacionada ao uso de álcool e cigarro, sendo 4,7\% e $16,1 \%$ que possuíam apenas o $1^{\circ}$ grau eram alcoolistas e fumantes, respectivamente. Estes dados estão de acordo com outros estudos populacionais. ${ }^{3}$ Assim como a pobreza está intimamente relacionada a vários comportamentos que afetam a saúde, neste estudo se observou que $17,4 \%$ e $4,9 \%$ dos idosos que possuíam uma renda mensal familiar de até dois salários eram fumantes e alcoolistas. No Reino Unido, por exemplo, os mais pobres fumam e bebem mais e têm uma dieta menos adequada. ${ }^{10,16} \mathrm{O}$ mesmo resultado foi encontrado em Goiânia para os fumantes. ${ }^{17}$ Silva et al. encontraram em seu estudo maior uso de álcool e "drogas ilícitas" no grupo que apresentava maior renda familiar mensal. ${ }^{18}$

A associação do alcoolismo e tabagismo com a ingestão alimentar neste grupo demonstrou que os idosos tinham uma média de uma a duas refeições diárias. Esse número de refeições por dia é considerado insuficiente para atender à demanda energética nesta população, visto que o número de refeições preconizado pelo guia alimentar para a população brasileira é de cinco a seis refeições diárias. ${ }^{19}$ São frequentemente encontradas carências graves de vitaminas e minerais em indivíduos que consomem uma elevada porcentagem de calorias na forma de álcool, bem como desnutrição energéticoprotéica, visto que no álcool há uma oferta de calorias vazias, o que sempre compromete a qualidade nutricional da dieta de alcoolistas. ${ }^{4}$

Alguns trabalhos têm demonstrado que o tipo de bebida alcoólica ingerida pode ser um fator que influencia os níveis séricos de homocisteína, 
vitaminas e ácido fólico. ${ }^{20}$ Recente estudo sugeriu que o alcoolismo crônico está acompanhado por perturbação do metabolismo de aminoácidos sulfurados e que a hiper-homocisteinemia etanolinduzida através de aguardente de cana-de-açúcar pode ser acompanhada de níveis séricos baixos de folato, agravando o estado nutricional destes pacientes. ${ }^{21}$

Quanto ao tabagismo e sua implicação no consumo alimentar, tem sido demonstrado em estudos experimentais que a nicotina e a exposição à fumaça do cigarro podem levar à perda de peso e a diminuição do consumo alimentar. ${ }^{10}$

A relação entre o tabagismo e a ingestão alimentar é extremamente complexa. A nicotina além de altamente tóxica tem ação estimulante e também depressiva sobre o sistema nervoso central, cujos efeitos são mediados pela liberação de catecolaminas, influenciando na regulação do apetite e ou no comportamento alimentar. ${ }^{17} \mathrm{O}$ estudo mostrou que $13,3 \%$ e $46,7 \%$ dos idosos que não tem relação familiar, são alcoolistas e tabagistas, respectivamente, sendo que $18,1 \%$ dos tabagistas vivem sozinhos. Estes dados levam a crer que ser usuário de álcool e fumo pode levar as pessoas a se afastarem umas das outras. Uma pessoa solitária tende a beber cada vez mais como meio de fugir da realidade e afastar a solidão. Em estudo realizado com jovens estudantes universitários, usuários de álcool e tabaco apresentaram maior satisfação em relação à frequência de lazer fora de casa do que nãousuários. ${ }^{18} \mathrm{~A}$ presença de um grupo familiar estável, seguro e de confiança é fundamental para manter boas relações familiares e evitar perdas em todos os sentidos, envolvendo bem-estar físico, social e emocional.
O presente estudo possui limitações metodológicas, entre as quais podemos citar: (1) como se trata de um estudo transversal, não é possível definir com precisão o que é causa e o que é efeito, para tais comprovações são necessários estudos longitudinais; (2) destacam-se as perdas amostrais decorrentes da dificuldade de obter os dados completos dos pacientes; (3) mesmo garantindo o sigilo, alguns pacientes podem não ter revelado o uso de álcool ou tabaco. Independentemente dessas limitações, os achados desta pesquisa corroboram os resultados encontrados na literatura.

\section{CONCLUSÃO}

Os idosos muitas vezes se tornam mais vulneráveis ao uso de álcool e tabaco, e isso aos poucos poderá gerar um grande problema populacional, já que o número de idosos no mundo está aumentando. Eles fazem parte de uma classe que apresenta vários problemas de saúde, uso de muitos medicamentos, que, combinados com as substâncias nocivas presentes no fumo e álcool, tornam os idosos mais susceptíveis a interações, agravamento do quadro instalado, dificuldade de recuperação e interação social.

Apesar de existirem vários estudos sobre o tabagismo e alcoolismo, ainda são poucos os que investigam as tendências e consequências desses hábitos entre a população idosa. Ações de saúde, políticas de prevenção e intervenção devem ser planejadas, utilizando uma abordagem integrada com o social, o psicológico e o biológico, visando a diminuir o consumo abusivo do álcool e o tabagismo, bem como prevenir seus malefícios à saúde. 


\section{REFERÊNCIAS}

1. Garrido R, Menezes PR. O Brasil está envelhecendo: boas e más notícias por uma perspectiva epidemiológica. Rev Bras Psiquiatr. 2002 abr.; 24 (suppl 1): 3-6.

2. Campos MTFS, Monteiro JBR, Ornelas APRC. Fatores que afetam o consumo alimentar e a nutrição do idoso. Rev Nutr. 2000 set./dez.; 13(3): 157-65.

3. Costa JSD, et al. Consumo abusivo de álcool e fatores associados: estudo de base populacional. Rev Saúde Pública 2004; 38(2): 284-91.

4. Moreira PFP, Martiniano Filho F. Aspectos nutricionais e o abuso do álcool em idosos. Envelhecimento e Saúde 2008; 14(1): 23-6.

5. Pinsky I, Jundi SARJE. O impacto da publicidade de bebidas alcoólicas sobre o consumo entre jovens: revisão da literatura internacional. Rev Bras Psiquiatr 2008; 30(4): 362-74.

6. Burns DM. Epidemiology of smoking-induced cardiovascular disease. Prog Cardiovasc Dis 2003 Jul./Aug.; 46(1): 11-29.

7. Glass JM, et al. Smoking is associated with neurocognitive deficits in alcoholism. Drug Alcohol Depend 2006 Apr.; 82(2): 119-26.

8. Nunes E. Consumo de tabaco: efeitos na saúde. Rev Port Clin Geral 2006; 22: 225-44.

9. Thomas GA, Rhodes J, Ingram JR. Mechanisms of disease: nicotine : a review of its actions in the context of gastrointestinal disease. Nat Clin Pract Gastroenterol Hepatol. 2005 Nov.; 2(11): 536-44.

10. Chiolero A, et al. Consequences of smoking for body weight, body fat distribution, and insulin resistance. Am J Clin Nut. 2008; 87: 801-9.

11. Blay SL, et al. Correlates of lifetime alcohol misuse among older community residents in Brazil. Int Psychogeriatr 2009 Apr.; 21(2): 384-91.

12. Barros MBA, et al. Prevalence of alcohol abuse and associated factors in a population based study. Rev Saúde Pública 2007 aug.; 41(4): 502-9.
13. Kim O, Baik S. Alcohol consumption, cigarette smoking, and subjective health in Korean elderly men. Addictive Behaviours 2004 nov.; 29(8): 1595-603.

14. Elbreder MF, et al. Perfil de mulheres usuárias de álcool em ambulatório especializado em dependência química. J Bras Psiquiatr 2008; 57(1): 9-15.

15. Peixoto SV, Firmo JOA, Lima-Costa MF. Condições de saúde e tabagismo entre idosos residentes em duas comunidades brasileiras (Projetos Bambuí e Belo Horizonte). Cad Saúde Pública 2006 set.; 22(9): 1925-34.

16. Feliciano AB, Moraes AS, Freitas ICM. O perfil do idoso de baixa renda no Município de São Carlos: um estudo epidemiológico. Cad Saúde Pública 2004 nov./dez.; 20(6): 1575-85.

17. Martins KA. Perfil de consumo alimentar de pacientes tabagistas em tratamento, na cidade de Goiânia, em 2006 . Dissertação[ Mestrado em Ciências da Saúde]— Rede Convênio CentroOeste (UnB, UFG e UFMS); 2006. 143p. [acesso 09 set 2009 ]; . Disponível em: http:// bdtd.bce.unb.br/tedesimplificado/tde_busca/ arquivo. php? codArquivo $=2096$.

18. Silva LVER, et al. Fatores associados ao consumo de álcool e drogas entre estudantes universitários. Rev Saúde Pública 2006; 40(2): 280-8.

19. Ministério da Saúde( Brasil). Secretaria de Atenção à Saúde. Coordenação Geral da Política de Alimentação e Nutrição: guia alimentar para a população brasileira. Brasília: MS; 2006. p.1-210.

20. Van der Gaag MS, et al. Effects of consumption of red wine, spirits, and beer on serum homocysteine. Lancet 2000 Apr.; 355: 1522.

21. Prioste RN, et al. Alterações no metabolismo da homocisteína induzidas por aguardente de cana-de-açúcar em alcoólatras. J. Bras. Patol. Med. Lab. 2003; 39(3): 203-6. 
